\title{
MRT Methodologies for Real-Time Simulation of Nonproliferation and Safeguards Problems
}

\author{
Alireza Haghighat ${ }^{1}$, Virginia Tech, haghighat@ vt.edu \\ Katherine Royston, Virginia Tech, royston@vt.edu \\ William Walters, Virginia Tech, walterswj@vt.edu \\ Nuclear Engineering Program, Mechanical Engineering Department, Virginia Tech \\ $900 \mathrm{~N}$ Glebe Road, Arlington, VA, USA
}

\begin{abstract}
This paper discusses advanced radiation transport methodologies developed by the Virginia Tech Transport Theory Group $\left(\mathrm{VT}^{3} \mathrm{G}\right)$ for real-time simulations. These methodologies can be referred to as Multi-stage Response-function particle Transport (MRT) methods in which the problem of interest is partitioned into several stages that each can be represented by a response function or set of coefficients. These stages are combined into a linear system of equations which are solved iteratively using the pre-calculated databases of response functions and/or coefficients. These databases are obtained by performing a set of "forward" and/or "adjoint" transport calculations for different conditions, e.g., material and geometry, by the appropriate Monte Carlo and deterministic code systems. This paper demonstrates the MRT method through two real-life problems including inspection of a spent nuclear fuel pool for safeguard applications and simulation of an active interrogation system for a cargo container for nonproliferation and security applications. It is demonstrated that the method provides the capability of performing realtime simulations while preserving the accuracy of highly time-consuming transport calculations.
\end{abstract}

\section{Introduction}

Over the past 60 years, there have been significant efforts devoted to development of accurate and fast methodologies and numerical techniques for solving particle transport problems in nuclear systems. The main approaches are deterministic [1] and Monte Carlo [2]. In the deterministic approach, the integro-differential form that is commonly used for fixed-source problems is solved via the discrete ordinates $(\mathrm{Sn})$ technique $[3,4]$ and the integral form that is commonly used for eigenvalue problems is solved via the method of characteristics (MOC) [5]. It is important to note that, for energy treatment, all the deterministic methods use a set of multigroup cross sections. Both deterministic methods are effective, however, they may suffer from large computer memory requirements, discretization approximations, and a slow rate of convergence. The Monte Carlo method has no requirement for discretization, and is effectively exact in representation of geometry and energy, however it is highly time-consuming for achieving statistically reliable results. Significant efforts are dedicated to the development of variance reduction techniques, which are capable of obtaining results for specific objectives. The method, however, may have large memory and time requirements if a large amount of information is needed.

Over the past 28 years, the first author and his students (including co-authors on this paper) have worked on new formulations for solving particle transport problems using deterministic and Monte Carlo methods and their combinations. For the deterministic transport equation, parallel and vector formulations, adaptive differencing formulations, acceleration techniques, automated techniques for multigroup cross-section generation, and pre- and post-processing software have been developed and used for various benchmarking and real-world problems encountered in nuclear power, nuclear nonproliferation and

${ }^{1}$ Corresponding author 
safeguards, and radiation therapy and diagnostics. These activities have resulted in the development of the PENTRAN code system [6], PENMSH and PENMSHXP [7], TITAN hybrid code system [5], Adaptive differencing Strategy (ADS) [8], spatial and angular multigrid and synthetic acceleration schemes [9]. In Monte Carlo particle transport, their efforts have resulted in the development of the automated variance reduction technique, i.e., CADIS (Consistent Adjoint Driven Importance Sampling) [10], and its implementation in the MCNP code system, i.e., $\mathrm{A}^{3}$ MCNP code [11]. The CADIS methodology has been used by various research groups in the US and worldwide $[12,13]$, as the methodology provides the ability for performing precise Monte Carlo simulations for difficult problems in a short time.

Although the aforementioned efforts have resulted in fast and accurate solution methods, they cannot yield solutions for most real-world problems in real-time. To achieve real-time solutions, it is necessary to carefully examine the problem physics, identify potential for partitioning the problem into calculation stages, i.e., multi-stage, and develop response methods based on the "forward" and "adjoint" particle transport formulations. In short, these techniques are referred to as "Multi-stage Response-function Transport (MRT)" methodologies. To describe the MRT approach we consider two problems related to nuclear safeguards and nuclear nonproliferation.

The remainder of this paper is organized as follows. Section 2 presents the MRT method for inspection of a spent fuel pool, and Section 3 presents the MRT method for active interrogation of a cargo container. Section 4 provides concluding remarks and future work.

\section{MRT formulation for Spent Nuclear Fuel Safeguards}

We have developed an MRT methodology, algorithm, and tool referred to as INSPCT-s (Inspection of Nuclear Spent fuel-Pool Calculation Tool ver. Spreadsheet) $[14,15]$ that uses computational and experimental results to identify potential fuel diversion in the Atucha-1 spent fuel pool, shown in Fig. 1.

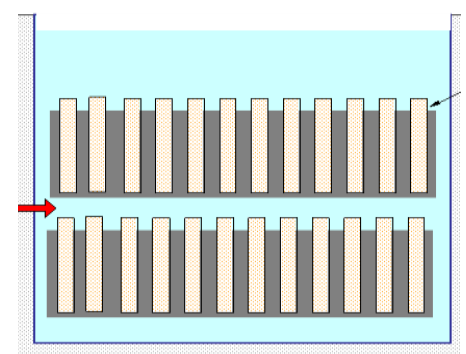

Fig. 1 - Schematic of the Atucha-1 Spent Fuel Pool

Since the Atucha-1 pool contains low-burnup fuel with long cooling times, it has low gamma source strength, hence, neutron detectors are employed for detection of possible fuel diversion for safeguards applications.

We have developed a MRT algorithm based on the adjoint function methodology [1] to determine response of a detector by

Where, $<>$ are Dirac signs referring to integration over all independent variables (space, energy and direction), $R$ refers to a detector response, $S$ refers to source particles (e.g., neutrons), and $\phi^{+}$refers to the importance function for a given detector.

To determine the detector response, it is necessary to determine neutron source and the importance function as a function of different parameters. The source is comprised of intrinsic source due to decay processes, i.e. $(\alpha, n)$ and spontaneous fission, and the fission 
neutrons due to the subcritical multiplication. To determine the latter, we employed the fission matrix (FM) approach which solves for fission neutrons by solving a system of linear equations using pre-calculated coefficient matrix elements. Hence, we developed a three-stage MRT methodology including: i) generation of intrinsic source; ii) generation of FM coefficients based on the intrinsic source for subcritical calculations; iii) generation of detector importance function for transport of neutron source to the detector. Stages one and two are coupled, and the resulting source distribution is used in Eq. 1 to obtain the detector response. The parameters for all stages can be interpolated to any value in the database.

A database of intrinsic source is generated for different parameters (burnup, enrichment, fuel lattice, and cooling time) using the ORIGEN-ARP code [16]. For the subcritical multiplication, a fission matrix (FM) approach is considered based on the following formulation

$$
F_{i}=\sum_{j=1}^{N}\left(a_{i, j} F_{j}+b_{i, j} S_{j}\right),
$$

where $F_{i}$ refers to fission neutrons generated in cell $i$ due to fission and source neutrons in cells $j=1, N, a_{i, j}$ refers to FM elements due to fission neutrons, and $b_{i, j}$ refers to FM elements due to intrinsic neutron sources. For the Atucha-1 pool, the cells refer to assemblies. We have demonstrated that the coefficients are highly localized and that $a$ and $b$ coefficients are similar. Further, our analyses demonstrated that only three sets of coefficients are needed depending on the assembly location including corner, center and side of the pool. Based on these considerations, a database of FM coefficients as a function of burnup, cooling time, enrichment and lattice structure was generated, and used in the simplified version of Eq. 2 given by

$$
F_{i}=\sum_{j=1}^{N} a_{i, j}\left(F_{j}+S_{j}\right),
$$

to obtain the fission neutron source due to subcritical multiplication. The coefficients are generated by performing a series of fixed source continuous-energy MCNP Monte Carlo [17] calculations.

To determine the adjoint function, the deterministic PENTRAN code system [6] was used for a neutron detector located in between fuel assemblies. Through analyses, it has been demonstrated that the field-of-view (FOV) of the detectors is small, and at most two rows of assemblies are "seen" by the detector as shown Fig. 2. This is very important conclusion, indicating the calculation volume is small, thereby resulting in short computation time and relatively easy generation of a database of adjoint-function distributions as a function of burnup, enrichment, cooling time, and lattice structure.

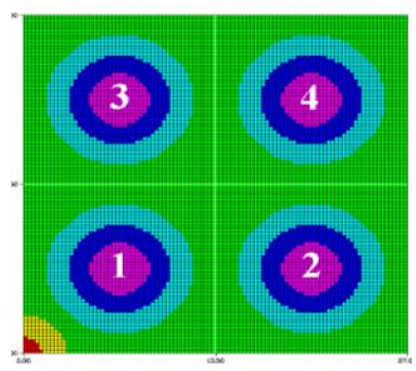

Fig. 2 - PENTRAN Adjoint Function Model

The INSPCT-s software was developed based on the 3-stage MRT methodology along with a Least Square Minimization (LSM) formulation to combine normalized measured and computed detector responses at several locations in the pool to determine 
potential fuel diversion within a given tolerance. INSPCT-s is implemented in an Excel 2003 spreadsheet using the Visual Basic for Applications (VBA) code to perform input and output processing. While the major portion of the calculations is performed using FORTRAN 95, the spreadsheet interface of the program makes the software user-friendly and allows for easy input, output, and visualization. Fig. 3 below gives a screen capture of the INSPCT-s software.

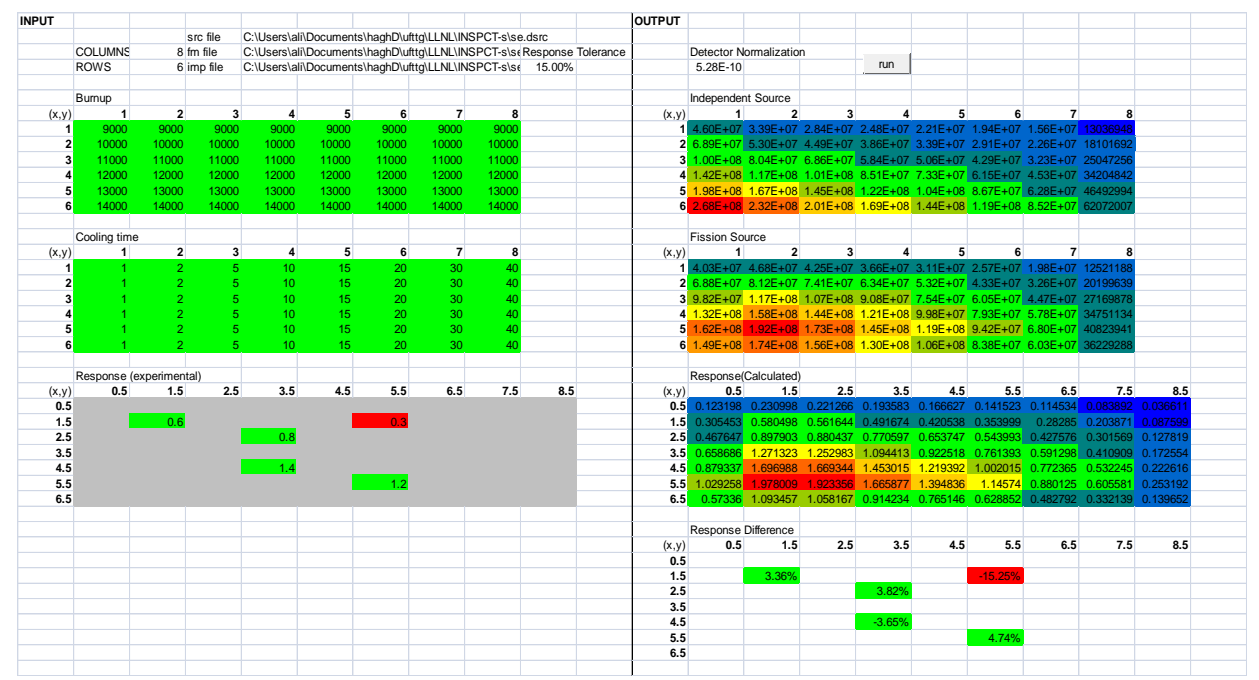

Fig. 3 - INSPCT-s Spreadsheet

The left-hand side of the above spreadsheet is allocated to the input including the names of the database files, tolerance, assembly-wise burnup distribution, assembly-wise cooling time distribution, and detector measurements. The right-hand side of the above spreadsheet provides the 'run' button and the output data including calculated intrinsic source, subcritical multiplication source, responses of detectors if they were placed in between fuel assemblies throughout the pool, and the relative differences and of the calculated and measured detector responses. In addition to the actual numerical values, colors are used for showing the strength of source distribution, detector response, and the relative differences of measured and calculated detector responses. The latter indication, if colored in red, informs an inspector of potential of fuel diversion.

Table 1 shows the burnup entries for a sample INSPCT-s calculation for an 8 by 6 assembly pool.

Table 1 - Sample Burnup Input for INSPCT-s

$\begin{array}{rrrrrrrrr}(\mathrm{x}, \mathrm{y}) & \mathbf{1} & \mathbf{2} & \mathbf{3} & \mathbf{4} & \mathbf{5} & \mathbf{6} & \mathbf{7} & \mathbf{8} \\ \mathbf{1} & 4000 & 4000 & 4000 & 4000 & 4000 & 4000 & 4000 & 4000 \\ \mathbf{2} & 5000 & 5000 & 5000 & 5000 & 5000 & 5000 & 5000 & 5000 \\ \mathbf{3} & 6000 & 6000 & 6000 & 6000 & 4000 & 6000 & 6000 & 6000 \\ \mathbf{4} & 7000 & 7000 & 7000 & 5000 & 7000 & 7000 & 7000 & 7000 \\ \mathbf{5} & 8000 & 8000 & 8000 & 8000 & 8000 & 8000 & 8000 & 8000 \\ \mathbf{6} & 9000 & 9000 & 9000 & 9000 & 9000 & 9000 & 9000 & 9000\end{array}$

Table 2 shows the corresponding cooling times. The green indicates that the values are within the databases, while the red indicates that these values lie outside and that coefficient values had to be extrapolated. 
Table 2 - Sample Cooling Time Input for INSPCT-s

$\begin{array}{rrrrrrrrr}(\mathrm{x}, \mathrm{y}) & \mathbf{1} & \mathbf{2} & \mathbf{3} & \mathbf{4} & \mathbf{5} & \mathbf{6} & \mathbf{7} & \mathbf{8} \\ \mathbf{1} & 1 & 2 & 5 & 10 & 15 & 20 & 30 & 45 \\ \mathbf{2} & 1 & 2 & 5 & 10 & 15 & 20 & 30 & 45 \\ \mathbf{3} & 1 & 2 & 5 & 10 & 15 & 20 & 30 & 35 \\ \mathbf{4} & 1 & 2 & 5 & 10 & 15 & 20 & 30 & 45 \\ \mathbf{5} & 1 & 2 & 5 & 10 & 15 & 20 & 30 & 5 \\ \mathbf{6} & 1 & 2 & 5 & 10 & 15 & 20 & 30 & 45\end{array}$

Table 3 shows the calculated detector response values. Note that the (x,y) values are in half units, which indicates that the detector is in between assemblies. The detector responses increase with burnup and decrease with cooling time and proximity to the edge of the pool, as would be expected.

Table 3 - Sample Detector Response Output for INSPCT-s

\begin{tabular}{|c|c|c|c|c|c|c|c|c|c|}
\hline$(x, y)$ & 0.5 & 1.5 & 2.5 & 3.5 & 4.5 & 5.5 & 6.5 & 7.5 & 8.5 \\
\hline 0.5 & 0.22 & 0.41 & 0.39 & 0.35 & 0.34 & 0.32 & 0.30 & 0.26 & 0.12 \\
\hline 1.5 & 0.61 & 1.13 & 1.06 & 0.96 & 0.88 & 0.82 & 0.77 & 0.65 & 0.30 \\
\hline 2.5 & 1.08 & 2.00 & 1.86 & 1.62 & 1.36 & 1.25 & 1.22 & 1.01 & 0.46 \\
\hline 3.5 & 1.74 & 3.23 & 2.99 & 2.41 & 2.00 & 1.94 & 1.80 & 1.46 & 0.67 \\
\hline 4.5 & 2.60 & 4.85 & 4.48 & 3.69 & 3.17 & 2.92 & 2.54 & 2.29 & 1.20 \\
\hline 5.5 & 3.33 & 6.16 & 5.65 & 4.84 & 4.17 & 3.60 & 3.02 & 2.59 & 1.30 \\
\hline 6.5 & 1.90 & 3.49 & 3.18 & 2.73 & 2.35 & 2.01 & 1.66 & 1.23 & 0.54 \\
\hline
\end{tabular}

INSPCT-s operates on any modern Windows laptop that can run Excel 2003 (or higher) in a fraction of second, and provides the capability of an inspector to examine different scenarios in a very short time. Note that the limiting factor here is the measurements. It is important to note that a certain amount computation time is needed for generating the three databases. For example for the current application, 4 hours were needed. Any condition that is within the range of applicability of the generated databases can be determined via a linear interpolation.

\section{Active interrogation simulation of SNM in cargo containers}

Under an NNSA funded project, in collaboration with Georgia Tech, we developed the AIMS-IFLEX (Active Interrogation for Monitoring of Special nuclear materials (SNMs) Incident Flux Expansion) toolkit, which is capable of simulating an active interrogation system for monitoring a cargo container with a hidden SNM region. Fig. 4 depicts a cargo container, containing a concealed SNM, e.g., a sphere of Highly Enriched Uranium (HEU), and a neutron source and gamma detector assembly for active interrogation of the container. For this demonstration, we have considered a D-T neutron source and CsI gamma detector. To develop an MTR method for this problem, the following stages are considered: i) D-T neutron source moderation and activation of cargo materials including any subcritical multiplication; ii) transport of fission neutrons throughout the container; iii) generation of gammas due to fission neutrons; iv) transport of gammas tor the detector face; and v) detection of gamma rays by the detector. These processes lead to a five-stage MRT methodology that is discussed below. This paper addresses Stages 1-4, which are developed by $\mathrm{VT}^{3} \mathrm{G}$, and Stage 5, which was developed by Georgia Tech, is discussed in Ref. 18 . 

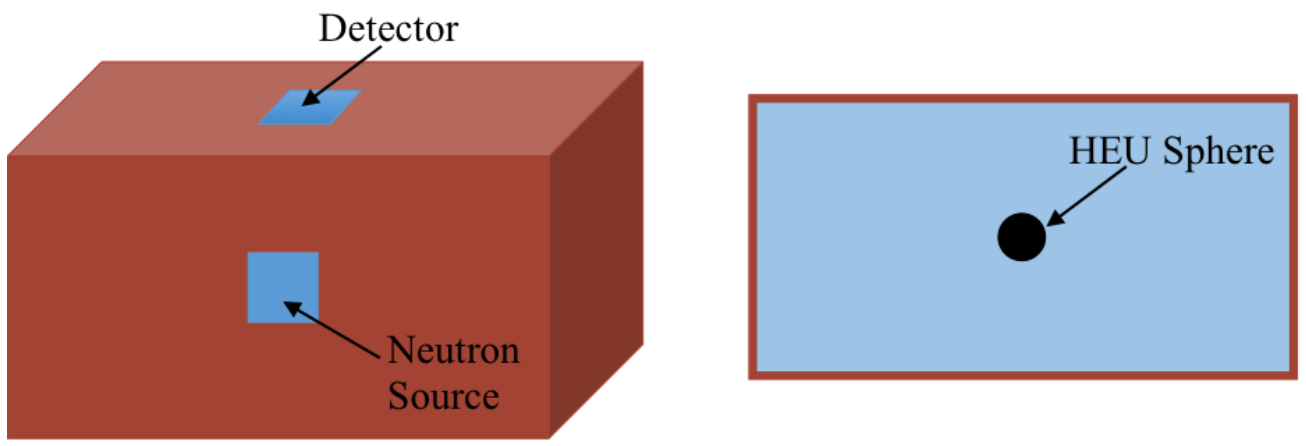

Scanning Direction

Fig. 4 - Cargo Container Model with Source-Detector Scanning

\subsection{Methodology}

This section introduces different methodologies used to simulate the first four stages of the processes that give the current of gamma rays at the face of the gamma detector shown in Fig. 4.

\subsubsection{Stage 1}

In Stage 1, we have developed a response-function methodology [19] to determine the fission density due to subcritical multiplication at the HEU site. The subcritical multiplication problem is split into several fixed source sub-problems, which are coupled through a set of response coefficients. The main variables solved for are shown in Fig. 5, where $S$ is the external neutron source, $J_{\text {in }}$ is the total integrated (over energy and area) current entering the Region of Interest (ROI), i.e., HEU sphere, $J_{\text {out }}$ is the total integrated current exiting the ROI, and $f$ is the fission rate in the ROI.

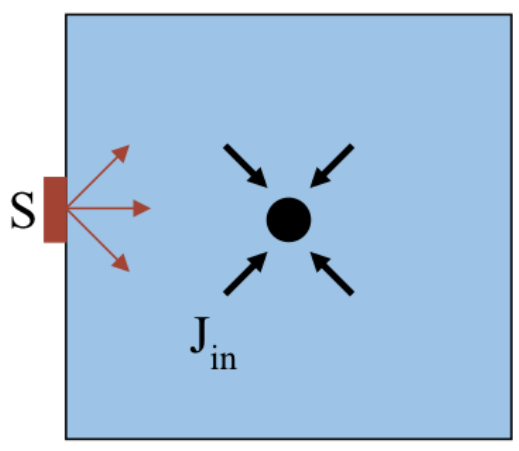

(a)

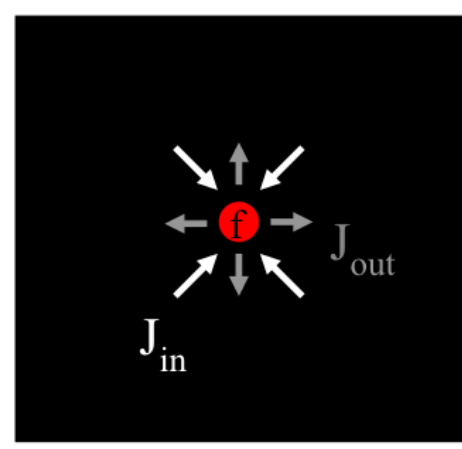

(b)

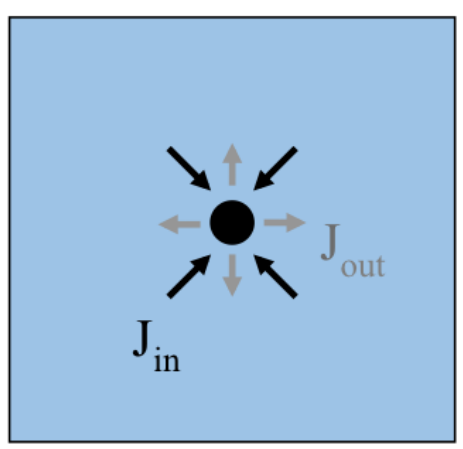

(c)

Fig. 5 - Calculation of neutron distribution due to subcritical multiplication by splitting into subproblems (a), (b), and (c).

In the first sub-problem, Fig. 5(a), only the source and cargo region are modeled (not the ROI), and so the source is $S$ and the incoming current to the ROI $\left(J_{i n}\right)$ is calculated. The corresponding response coefficient is given by

$$
\alpha_{\text {s,in }}=\frac{J_{\text {in }}}{S} \text {. }
$$

In the second sub-problem, Fig. 5(b), only the ROI is considered and the source is the incoming current $\left(J_{\text {in }}\right)$ from the first sub-problem. The outgoing current $\left(J_{\text {out }}\right)$ and the fission rate in the ROI $(f)$ are calculated and the following response coefficients are determined: $\alpha_{\text {in }, \text { out }}=J_{\text {out }} / J_{\text {in }}$ and $\alpha_{\text {in,f }}=f / J_{\text {in }}$. 
In the final sub-problem, Fig. 5(c), just the cargo region is considered again, but now the source is $J_{\text {out }}$ and the reflected current $\left(J_{\text {in }}\right)$ is calculated. This yields the response coefficient given by $\alpha_{\text {out,in }}=J_{\text {in }} / J_{\text {out }}$. Now, if we couple the three sub-problems, we can obtain a formulation for the total fission density expressed by

$$
f=\frac{S \alpha_{s, i n} \alpha_{i n, f}}{1-\alpha_{\text {in }, \text { out }} \alpha_{\text {out }, \text { in }}} .
$$

All response coefficients are calculated by performing fixed-source MCNP5 Monte Carlo calculations on each sub-problem and using the surface source write/read capability of MCNP5.

If the location of the ROI in the cargo is changed, only the $\alpha_{s, \text { in }}$ coefficient is expected to significantly change. This coefficient is assumed to behave like the flux a distance $r$ from a point source in a diffusive medium and is fit to

$$
\alpha_{s, i n}(\mathrm{r})=\frac{\alpha_{0}}{r} e^{-\sigma_{0} r}
$$

The constants $\alpha_{0}$ and $\sigma_{0}$ are found by calculating $\alpha_{s, \text { in }}$ for several ROI locations and using a least-squares regression to minimize the difference between data points.

\subsubsection{Stage 2}

In the $2^{\text {nd }}$ stage, the fission neutron flux distribution within the container due to the fission rate calculated by the $1^{\text {st }}$ stage is determined. An MCNP5 model of the cargo is created using a surface source into the ROI and the neutron flux is tallied at radial distances $r$ from the ROI. Calculations with the HEU in the ROI are used to obtain $\phi_{1}(r)$ and with regular cargo in the ROI to obtain $\phi_{2}(r)$. The group-dependent flux distribution difference per fission neutron is then

$$
\tilde{\phi}_{g}^{n}(\mathrm{r})=\frac{\phi_{g, 1}(r)-\phi_{g, 2}(r)}{f}
$$

This distribution can be interpolated to any radial distance $r$ using

$$
\tilde{\phi}_{g}^{n}(\mathrm{r})=\frac{a_{i}}{r} e^{-\sigma_{i} r},
$$

where $a_{i}$ and $\sigma_{i}$ are coefficients for each interpolation interval $i$. The neutron flux distribution due to fission can be found by multiplying the Eq. (8) result by the fission neutron rate $f$ from Eq. (5).

\subsubsection{Stage 3}

In the $3^{\text {rd }}$ stage, the gamma distribution due to $(n, \gamma)$ interaction of fission neutrons in the cargo is determined by

$$
S_{i, g}=\sum_{g^{\prime}} \phi_{i, g^{\prime}}^{n} \sigma(n, \gamma)_{i, g^{\prime} \rightarrow g}
$$

where $\phi_{i, g}^{n}$ is the fission neutron flux distribution obtained from $2^{\text {nd }}$ stage and $\sigma_{i, g^{\prime} \rightarrow g}$ is the Bugle-96 $(n, \gamma)$ cross sections [20] from neutron energy group $g^{\prime}$ to gamma energy group $g$. 


\subsubsection{Stage 4}

In the $4^{\text {th }}$ stage, using the importance function methodology (Eq. 1), we determined the gamma current at the detector window due to fission neutrons by [21]

$$
J^{+}\left(\vec{r}, \mathrm{E}_{\mathrm{g}}\right)=\sum_{i} \sum_{g^{\prime}} \phi_{i, g^{\prime}}^{\dagger} S_{i, g^{\prime}} V_{i},
$$

where $i$ and $g$ refer to spatial mesh and energy group, respectively, $J^{+}$is the outgoing gamma current from the container, at the face of the detector for the $g^{\text {th }}$ energy group, $\phi^{\dagger}$ is adjoint function for the gamma detector, $S$ is gamma source distribution, and $V$ is spatial mesh volume. Note the adjoint function is determined based on a boundary value of $\tilde{\psi}_{g}^{\dagger}=\delta\left(E-E_{g}\right) \delta\left(\vec{r}-\vec{r}_{d}\right)$ for importance of gamma-rays entering the detector. The adjoint function is calculated for different groups and parameters by the TITAN deterministic transport code [5].

\subsection{AIMS software}

Based on the formulations described in Section 3.1 for the four stages of an active interrogation system, the AIMS (Active Interrogation Monitoring of Special-nuclearmaterials) software tool was developed. The AIMS software tool [22] is in the form of a script that uses the pre-calculated databases containing the necessary coefficients and distributions for Stages 1-4. The gamma current at the detector window is then output for different HEU locations and source-detector assembly offset positions specified by the user.

\subsection{Example results for a cargo container}

To test AIMS, a cargo container with the scanning source-detector assembly shown in Fig. 4 used. The reference model is a cargo container with dimensions of $244 \mathrm{~cm} \mathrm{x} 259 \mathrm{~cm}$ $\mathrm{x} 141 \mathrm{~cm}$. The neutron source is an isotropic D-T surface source $(14.1 \mathrm{MeV})$ of size $13.5 \mathrm{x}$ $13.5 \mathrm{~cm}^{2}$ and the detector window is $13.5 \times 13.5 \mathrm{~cm}^{2}$. A $25 \mathrm{~kg}$ sphere of HEU with a radius of $6.75 \mathrm{~cm}$ is located within the container.

To examine the capability/limitations of AIMS, the HEU sphere is simulated in the positions depicted in Fig. 6 and Table 4 and the source-detector assembly is moved along the length of the container. The source-detector assembly begins at the center of the cargo container, i.e., aligned with the HEU sphere along the z-axis, and then is offset by 10, 20, 30 , and $50 \mathrm{~cm}$. For demonstration in this paper, homogeneous third-density water $(0.33$ $\mathrm{g} / \mathrm{cm}^{3}$ ) cargo is considered Simulations have also been conducted for additional HEU positions and half-density water, wood, and steel cargo [22]. 


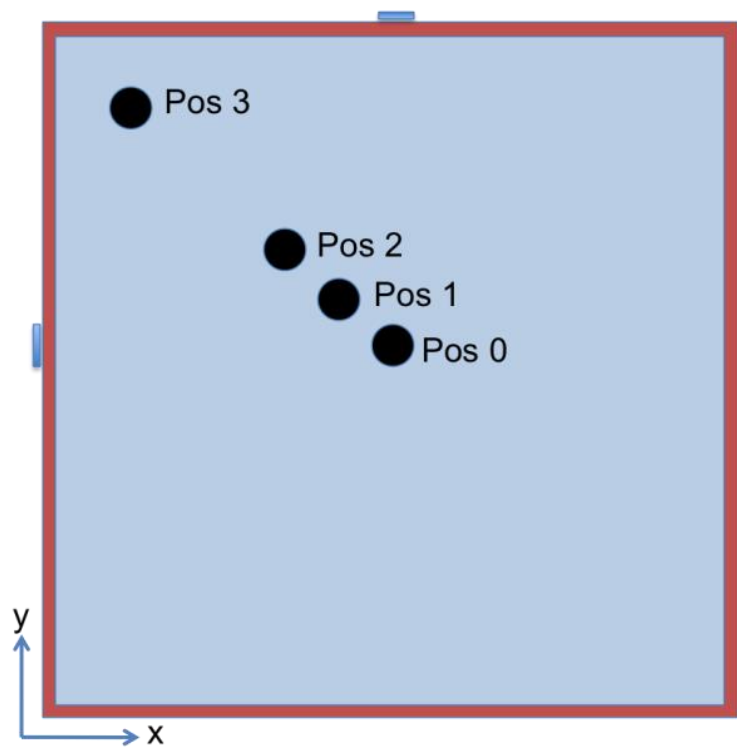

Table 4 - HEU position coordinates

\begin{tabular}{cc}
\hline Position & $(\mathbf{x}, \mathbf{y})$ \\
\hline $\mathbf{0}$ & $(0 \mathrm{~cm}, 0 \mathrm{~cm})$ \\
$\mathbf{1}$ & $(-20 \mathrm{~cm}, 20 \mathrm{~cm})$ \\
$\mathbf{2}$ & $(-40 \mathrm{~cm}, 40 \mathrm{~cm})$ \\
$\mathbf{3}$ & $(-100 \mathrm{~cm}, 100 \mathrm{~cm})$ \\
\hline
\end{tabular}

Fig. 6 - HEU positions simulated

Results for the calculation of fission density (Stage 1) in cargo consisting of thirddensity water by MCNP5 and AIMS are given in Table 5. Note that the same set of coefficients is used regardless of HEU position. Excellent agreement with the Monte Carlo reference solution is seen for HEU positions 0,1 , and 2. This agreement is reduced as the HEU approaches the container edge, i.e., position 3, because position specific response coefficients are needed when the HEU is near the container wall. With these new coefficients, the AIMS solution for position 3 is within 2\% of the MCNP5 reference fission density.

Table 5 - Fission density for different ROI positions in third-density water cargo calculated by MCNP5 and the response method

\begin{tabular}{cccc}
\hline ROI Position & MCNP5 (16) & AIMS & Difference \\
\hline $\mathbf{0}$ & $5.55 \times 10^{-3}(0.09 \%)$ & $5.60 \times 10^{-3}$ & $1.0 \%$ \\
$\mathbf{1}$ & $1.07 \times 10^{-2}(0.09 \%)$ & $1.05 \times 10^{-2}$ & $-1.9 \%$ \\
$\mathbf{3}$ & $1.72 \times 10^{-2}(0.15 \%)$ & $1.66 \times 10^{-2}$ & $-3.2 \%$ \\
$\mathbf{3}$ (modified)* & $7.18 \times 10^{-3}(0.08 \%)$ & $1.08 \times 10^{-2}$ & $50.3 \%$ \\
*Coefficients for position 3 are reevaluated, considering that HEU is placed at this \\
position
\end{tabular}

The 2-3 MeV gamma current at the detector window for a source-detector assembly scanning the container is calculated using AIMS and MCNP5 for third-density water cargo in Fig. 7 - Current at detector for different HEU positions for the HEU positions in Fig. 6. In Fig. 7(a), the position 0 and 1 AIMS results are within the uncertainty of the MCNP5 reference solutions for all scanning positions. Differences of $8-13 \%$ are found relative to the MCNP5 for HEU position 2. In Fig. 7(b), the HEU is approaching the container wall, which results in consistently higher AIMS solutions compared with MCNP5. Note that, as the HEU nears the container wall, passive detection may be possible. If position specific response coefficients (PSC) are used for HEU positions 3, the AIMS solutions are within $1 \sigma$ of the MCNP5 reference solutions. 


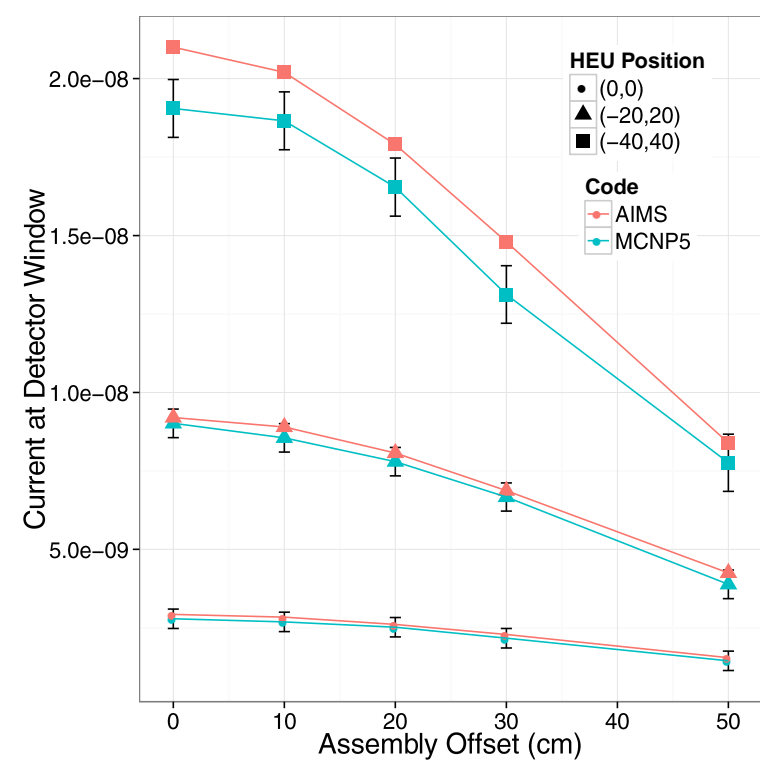

(a) HEU positions 0,1 , and 2

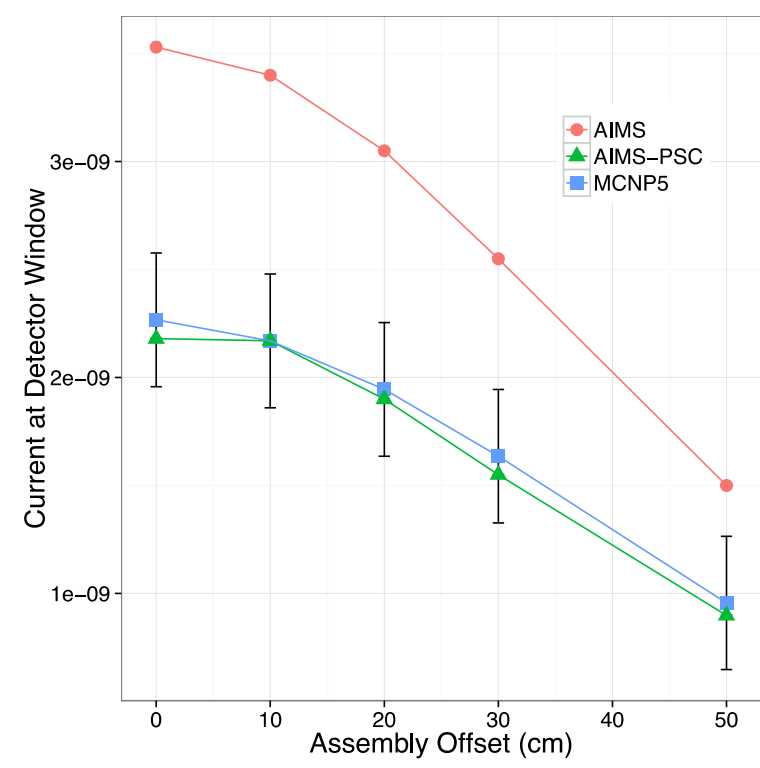

(b) HEU position 3

Fig. 7 - Current at detector for different HEU positions where AIMS-PSC refers to AIMS with position specific coefficients

AIMS has the advantage of being able to accurately predict the gamma current at the detector in significantly less time than a simulation completely done in MCNP5. The computation time to generate the pre-calculated database used by AIMS for this problem is given in Table 6.

Table 6 - Computation time for AIMS database

\begin{tabular}{cc}
\hline Pre-calculated value & Computation Time \\
\hline Response coefficients (serial) & 60 hours \\
Group $8(2-3 \mathrm{MeV})$ adjoint function $(8$ proc.) & 6 hours \\
\hline
\end{tabular}

Table 7 compares AIMS and MCNP5 computation times for determination of the gamma current for 25 cases including 6 HEU locations and 5 scanning positions for each HEU location. Table 7 indicates that AIMS indeed can achieve accurate solutions in the order of minutes, while the straightforward Monte Carlo transport calculations require months/years of computation time.

Table 7 - Computation times for AIMS and MCNP5

\begin{tabular}{ccc}
\hline \multicolumn{2}{c}{ Simulation Case } & Computation Time \\
\hline \multicolumn{2}{c}{ AIMS for all HEU positions (serial) } & 0.06 hours \\
\multirow{2}{*}{ MCNP5 $^{*}$} & HEU position 0 or 3 & 495 hours \\
(8 processors) & HEU position 1 & 254 hours \\
& HEU position 2 & 63 hours \\
\hline
\end{tabular}

*Times are for the MCNP5 uncertainties shown in figures (4.7\%-32.3\%)

\section{Concluding remarks and future work}

This paper introduces Multi-stage Response-function particle Transport (MRT) methodologies for solving real-life problems, accurately and in real-time. MRT algorithms 
have been developed for two real-time problems including inspection of a spent nuclear fuel pool for safeguard applications and simulation of an active interrogation system for a cargo container for nonproliferation and security applications.

The INSPCT-s software has been developed based on an MRT algorithm and can examine different scenarios in orders of seconds as compared to hours of computation with standard transport codes using parallel computers. Actually, its limiting factor is the time to acquire measurements. It is important to note that a certain amount of one-time computation is needed for generating the three databases. For example, for the current application, 4 hours were needed.

The AIMS software has been developed based on an MRT algorithm and can simulate scanning of a cargo container by a source-detector assembly. It is demonstrated that AIMS can yield information within a very short time, several orders of magnitude faster the standard transport codes. For example, for scanning 30 cases, AIMS yields the gamma current at the detector window in orders of minutes, while the MCNP Monte Carlo calculations require months or years of computation time.

Future work should extend the aforementioned formulations and tools for determination of axially-dependent pin-wise fission density and material composition in the spent fuel, and develop novel formulations for containers with heterogeneous cargo. Experimental benchmarks are needed for evaluation of the capabilities and limitations of the INSPCT-s and AIMS software tool.

\section{Acknowledgements}

Funding for the development of INSPCT-s methodology and software was provided by the Lawrence Livermore National Lab, and the funding for the development AIMS methodology and software was provided by the National Nuclear Security Administration (Contract No. DE-FG-52-09NA29360)

\section{References}

1. G. I. Bell and S. Glasstone, Nuclear Reactor Theory, Robert E. Krieger Publishing, Malabar, FL, 1985.

2. A. Haghighat, Monte Carlo Methods for Particle Transport, CRC Press Taylor \& Francis Group, Boca Raton, FL, 2014.

3. E. E. Lewis and W. F. Miller, Computation Methods of Neutron Transport, John Wiley \& Sons, New York, 1984.

4. R.J.J. Stammler and M.J. Abbate, Methods for Steady-State Reactor Physics in Nuclear Design, Academic Press, 1983.

5. C. Yi and A. Haghighat, A 3-D Block-Oriented Hybrid Discrete Ordinates and Characteristics Method. Nuclear Science and Engineering, 164(3), 2010.

6. G. E. Sjoden and A. Haghighat, PENTRAN - A 3-D Cartesian Parallel Sn Code with Angular, Energy, and Spatial Decomposition, Proceedings of the Joint Int. Conf. on M\&C and Supercomputing in Nuclear Applications, Vol. II, 1997.

7. C. Yi and A. Haghighat, "PENMSH-XP: A 3-D Mesh Generator for PENTRAN," Version 1.5b, University of Florida (2008).

8. Haghighat, A., G.E. Sjoden, and V.N. Kucukboyaci, "Effectiveness of PENTRAN's Unique Numerics for Simulation of the Kobayashi Benchmarks," Progress in Nuclear Energy, 39(2), pp. 191-206, 2001.

9. V. Kucukboyaci and A. Haghighat, "A Simplified Angular Multigrid Method to Accelerate Sn Calculations," Trans. Am. Nucl. Soc., 81, pp. 140, 1999 ANS Winter Meeting, Nov. 14-18, 1999, Long Beach, CA. 
10. J. C. Wagner and A. Haghighat, "Automated variance reduction of Monte Carlo shielding calculations using the discrete ordinates adjoint function," Nuclear Science and Engineering, 128 (2), pp. 186, 1998.

11. A. Haghighat, and J. C. Wagner, "Monte Carlo variance reduction with deterministic importance functions," Progress of Nuclear Energy Journal, 42 (1), pp. 25, 2003.

12. J. C. Wagner, D. E. Peplow, S. W. Mosher, and T. M. Evans, "Review of Hybrid (Deterministic/ Monte Carlo) Radiation Transport Methods, Codes, and Applications at Oak Ridge National Lab, Progress in Nuclear Science and Technology, 2, 808-814, 2010.

13. J. C. Wagner, D. E. Peplow, and S. W. Mosher, "FW-CADIS Method for Global and Regional Variance Reduction of Monte Carlo Radiation Transport Calculations," Nuclear Science and Engineering, 176, 37-57, 2014.

14. W. Walters, A. Haghighat, M. Wenner, Y. Ham, and S. Sitaraman, "A Methodology for Determination of Detector Response for Inspection of a Spent Fuel Pool," Proc. PHYSOR 2010, Pittsburgh, PA, May 9-14, 2010.

15. W. Walters, W. A. Haghighat, S. Sitaraman and Y. Ham, "Development of INSPCT-s for Inspection of Spent Fuel Pool," Journal of ASTM International, 9(4), 2012.

16. "SCALE, A Comprehensive Modeling and Simulation Suite for Nuclear Safety Analysis and Design," ORNL/TM-2005/39, Version 6.1, Oak Ridge National Laboratory, Oak Ridge, TN, 2011.

17. X-5 Monte Carlo Team, "MCNP-A General Monte Carlo Code for Neutron and Photon Transport, Version 5," Los Alamos National Laboratory, 2003.

18. T. Zipperer, F. Rahnema, and D. Zhang, "Pulse height tally response expansion method for application in detector problems," Int. J. Mod. Phys. Conf. Ser., 27 (2014).

19. W. Walters, A. Haghighat, K. Royston, C. Yi and G. Sjoden, "A Response Function Methodology to Calculate Induced Fission Neutron Flux Distribution in an Active Interrogation System," Progress in Nuclear Science and Technology, 4, pp. 533, 2014.

20. "BUGLE-96: Coupled 47 Neutron, 20 Gamma-Ray Group Cross Section Library Derived from ENDF/B-VI for LWR Shielding and Pressure Vessel Dosimetry Applications," DLC-76, Oak Ridge National Laboratory (1996).

21. K. Royston, A. Haghighat, W. Walters, C. Yi, and G. Sjoden, "Determination of current at a detector window using a hybrid adjoint function methodology," Progress in Nuclear Science and Technology, 4, pp. 528, 2014.

22. A. Haghighat, K. Royston, W. Walters, C. Yi, and G. Sjoden, "Development of AIMS Hybrid Tool for Active Interrogation," INMM 54 Annual Meeting, Palm Desert, CA, July 14-18, 2013. 